

Die AG Young DOG richtet sich an junge Assistenzärzte und Nachwuchswissenschaftler. Sie fokussiert ihre Aktivitäten auf die Förderung des wissenschaftlichen Nachwuchses in der Ophthalmologie und die Vereinbarkeit von Klinik, Forschung und Familie.

Die Mitgliedschaft in der AG ist den Mitgliedern der DOG vorbehalten. Mitglied werden können Assistenzärzte, habilitierte Ärzte und Wissenschaftler sowie Professoren bis W 2.

Nähere Information zur AG Young DOG finden Sie unter http://www. dog.org/?cat=137

\section{Deniz Hos}

Zentrum für Augenheilkunde, Uniklinik Köln, Köln, Deutschland

\section{Korrespondenzadresse:}

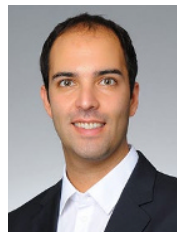

Dr. med. Dr. nat. med. Deniz Hos Zentrum für Augenheilkunde, Uniklinik Köln

Kerpener Str. 62

50924 Köln

deniz.hos@uk-koeln.de

Ophthalmologe 2018 $\cdot 115: 530$ https://doi.org/10.1007/s00347-0180721-1

(c) Springer Medizin Verlag GmbH, ein Teil von Springer Nature 2018

\title{
Augenärztliche Weiterbildung in Deutschland
}

\section{Allgemeines}

Die Weiterbildung zum Facharzt für Augenheilkunde beginnt nach der Approbation im Rahmen der ärztlichen Berufstätigkeit. Dies hat unter Anleitung von zur Weiterbildung befugten Ärzten in einer zugelassenen Weiterbildungsstätte zu erfolgen. Weiterbildungsbefugte haben eine befristete Zulassung durch die Landesärztekammer, wobei die Dauer der Befugnis vom jeweiligen Patientenspektrum und den Fallzahlen abhängt und zwischen 12 und 60 Monaten beträgt. Weiterbildungsstätten können Universitätskliniken, periphere Krankenhäuser, medizinische Versorgungszentren oder Praxen sein. Die Weiterbildungszeit zum Facharzt für Augenheilkunde beträgt 60 Monate, wobei bis zu 36 Monate im ambulanten Bereich abgeleistet werden können. Eine Anrechnung von Weiterbildungszeiten aus anderen Fachdisziplinen ist nicht möglich.

\section{Weiterbildungsordnung}

Die Weiterbildungsordnung liegt in der Verantwortung der jeweiligen Landesärztekammer. Aktuell richten sich alle Landesärztekammern nach der Weiterbildungsordnung der Bundesärztekammer. Die Weiterbildungsrichtlinien enthalten die Weiterbildungsinhalte und sog. Richtzahlen. Die Richtzahlen definieren die Menge an Untersuchungs- und Behandlungsverfahren, die erreicht werden müssen. Beispiele hierfür sind: Durchführung und Befundung von Fluoreszenzangiographien: 50; Lokal- und Regionalanästhesien: 100; laserchirurgische Eingriffe an der Netzhaut: 100; Augenmus- keloperationen: 10; intraokulare Eingriffe: 25. Die pro Weiterbildungsjahr erreichte Richtzahl muss (jährlich!) dokumentiert und durch den Weiterbilder abgezeichnet werden. Außerdem ist eine jährliche Dokumentation eines Weiterbildungsgesprächs zwischen dem Antragsteller und dem Weiterbilder erforderlich, in welchem der Gesprächsinhalt (bisheriger Verlauf der Weiterbildung, künftige Ziele) dokumentiert wird.

\section{Anerkennung von Teil-/Fehl-/ \\ Forschungszeiten}

Damit ein Weiterbildungsabschnitt angerechnet werden kann, ist eine Beschäftigung von mindestens 6 Monaten notwendig. Eine Weiterbildung in Teilzeit (hierbei ist eine Beschäftigungszeit von mindestens $50 \%$ einer Vollbeschäftigung notwendig) ist nach Antragstellung möglich, wobei die Weiterbildungszeit entsprechend verlängert werden muss. Fehlzeiten von mehr als 6 Wochen müssen nachgearbeitet werden. Forschungszeiten werden in der Regel nicht anerkannt, wobei eine Anerkennung in Einzelfällen nach (!) Antragstellung möglich ist. In diesem Zusammenhang hat beispielsweise die Landesärztekammer Westfalen-Lippe ein Modellprojekt für die Anerkennung von Forschungszeiten in der Weiterbildung entwickelt. Hier konnten bereits mehreren Teilnehmern Forschungszeiten auf die Weiterbildungszeit angerechnet werden [1].
Weiterbildung in Theorie und Praxis

Aktuell gibt es kein einheitlich geregeltes Weiterbildungscurriculum in der Augenheilkunde, und es besteht eine große Variation zwischen den Weiterbildungscurricula der Weiterbilder. Zur Analyse der Weiterbildungssituation in Deutschland hat die AG Young DOG 2014 und 2016 eine Umfrage unter allen DOG-Mitgliedern mit „Assistenzarztstatus“ durchgeführt [2,3]. In beiden Umfragen gaben die Befragten an, dass es in etwa $60 \%$ der Fälle kein strukturiertes Weiterbildungscurriculum in der Weiterbildungsstätte gibt und dass in nur knapp 20-30\% der Fälle die Anforderungen im Weiterbildungskatalog vollständig erfüllt werden, während in 60 $70 \%$ der Fälle die Anforderungen nicht vollständig erfüllt sind. Diese Zahlen sind alarmierend und sollten von allen Beteiligten zum Anlass genommen werden, ein strukturiertes und verbindliches Weiterbildungscurriculum zu etablieren, um die Attraktivität unseres Fachs langfristig aufrechtzuerhalten und eine Patientenversorgung auf hohem Niveau zu gewährleisten.

\section{Literatur}

1. Joachim S (2017) Ein Blick über den Tellerrand - Forschung, Weiterbildung und Kommunikation. Karger Kompass Ophthalmol 3:129-130. https:// doi.org/10.1159/000477082

2. Hos D, Steven P, Dietrich-Ntoukas T (2015) The situation of residents in ophthalmology in Germany: Results of an online survey. Ophthalmologe 112(6):498-503. https:// doi.org/10.1007/s00347-015-0030-x

3. Prokosch-Willing V, Hos D, Joachim SC (2017) Research during residency. Ophthalmologe 114(10):901-905. https://doi.org/10.1007/s00347-0170566-z 